\title{
Steady State Heat Conduction in Cylinders With Multiple Continuous Line Heat Sources
}

\author{
B. A. Peavy \\ (February 1, 1963)
}

\begin{abstract}
A mathematical analysis is presented for steady state heat conduction in cylinders, consisting of one or two isotropic materials disposed in concentric cylindrical volumes around the axis, heated by one or more continuous line heat sources parallel to the axis. The analysis considers both cylinders of infinite length, and cylinders of finite length subject to longitudinal heat flows arising from end conditions. Numerical solutions are given considering the line sources to be equal in magnitude and equally spaced angularly at a uniform radius from the axis. An outgrowth of this analysis has been the consideration of several new methods for the determination of the thermal properties of materials.
\end{abstract}

\section{Introduction}

This paper presents a mathematical analysis of steady state heat conduction in a cylinder, consisting of one or two isotropic materials disposed in concentric cylindrical volumes around the axis, heated by one or more continuous line heat sources parallel to the axis. The analysis considers both cylinders of infinite length, and cylinders of finite length subject to longitudinal heat flows arising from end conditions. The length of the line heat sources equals the length of the cylinder.

Problems concerning a single continuous line heat source are treated by Carslaw and Jaeger [1]. ${ }^{1} \quad$ The methods used in their book for single sources give an insight useful for the solution of problems involving multiple sources. In this paper, the multiple line sources are considered to be equal in magnitude, and to be equally spaced angularly at a specific radius from the axis. However, it is possible by the method used to obtain solutions for multiple sources having various and differing magnitudes, radii, and angular positions.

This investigation was made in connection with an experimental steady state method [2] of determining the thermal conductivity of granular materials, using the arrangement shown

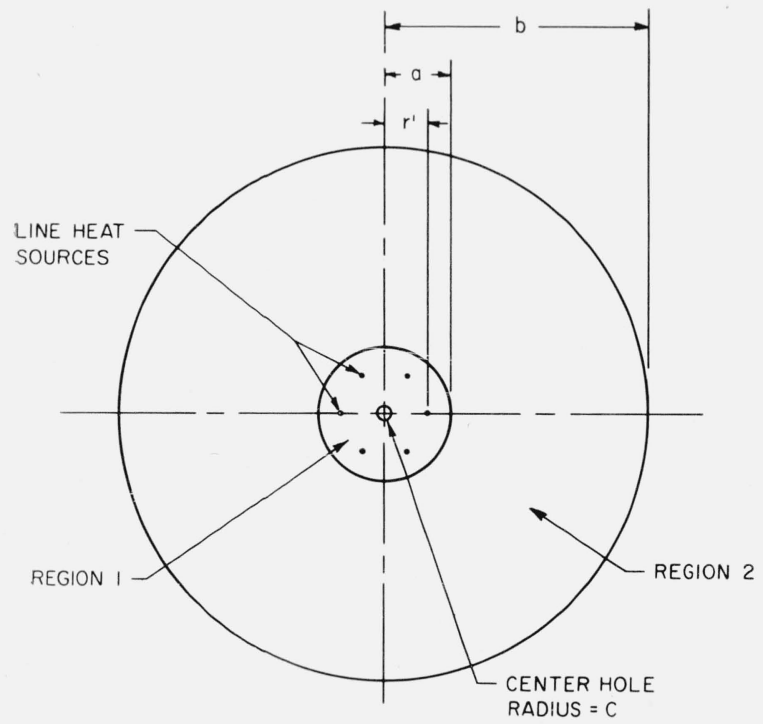

FiguRE 1. Cross section of two concentric cylinders with line heat sources in the inner cylinder. 
schematically in figure 1 . The granular material comprises region 2. A long cylindrical ceramic core (region 1) is heated electrically by resistance wires in the equally spaced small longitudinal holes of the core. A thermocouple in the central hole of the core indicates its temperature at the axis, from which the average core surface temperature is inferred. If there is no heat source or sink inside the region enclosed by the circle of heater wires and there is negligible contact resistance between the two regions, it is intuitive that the temperature in the hole of radius $c$ at the center of the core is equal to the average temperature at the radius passing through the circle of heater wires. The holes are assumed to be virtual line sources. On this basis, a simple expression can be written for the average temperature at the radius of the hole at the center of the core:

$$
\bar{\theta}_{c}=\bar{T}_{c}-T_{b}=\frac{m Q}{2 \pi k_{1}}\left[\ln a / r^{\prime}+\sigma \ln b / a\right]=\frac{m Q \ln b / a}{2 \pi k_{2}}\left[1+\frac{\ln a / r^{\prime}}{\sigma \ln b / a}\right]
$$

where

$$
\begin{aligned}
\bar{T}_{c} & =\text { temperature in hole of radius } c \text { at the center of the core, } \\
T_{b} & =\text { uniform temperature at radius, } r=b, \\
Q & =\text { heat input of line source per unit time per unit length, } \\
m & =\text { number of line sources, } \\
\sigma & =k_{1} / k_{2}, \\
k_{1} & =\text { thermal conductivity of inner cylinder material, } \\
k_{2} & =\text { thermal conductivity of outer cylinder material, } \\
a & =\text { outside radius of inner cylinder, } \\
b & =\text { outside radius of outer cylinder, } \\
r^{\prime} & =\text { radius passing through heat sources }\left(r^{\prime}<a\right) .
\end{aligned}
$$

If the other fixed quantities in it are known, eq (1) serves for the experimental determination of $\sigma$, or, if an adequate value $\sigma$ can be assumed, of $k_{2}$, the thermal conductivity of the material of the outer cylinder.

In this paper, eq (1) is derived analytically for cylinders of infinite length, and an examination is made of departures if the core temperature were measured not at the center of the core, but at a small radius and variable angular position. For cylinders of finite length, with temperatures measured at midlength, departures from eq (1) due to longitudinal heat flow caused by end effects are given for variations in $\sigma$ and cylinder length.

\section{Mathematical Development}

\subsection{Infinitely Long Cylinder}

\section{a. Single Line Heat Source}

Consider a single continuous line heat source of magnitude $Q$ heat units per unit time per unit length and infinite in length, located in an isotropic medium at radius $r^{\prime}$, polar angle $\varphi^{\prime}$. At a point in the medium, $(r, \varphi)$, the distance measured from the source is $R=\left[r^{2}+r^{\prime 2}-2 r r^{\prime} \cos \right.$ $\left.\left(\varphi-\varphi^{\prime}\right)\right]^{\frac{1}{2}}$. The heat flux from the source at a line of unit length parallel to the source must be satisfied by the limit

$$
\lim _{R \rightarrow 0}\left[-2 \pi k R \frac{d \theta}{d R}\right]=Q
$$

where $k$ is the thermal conductivity of the medium containing the source and $\theta$ is the temperature above an arbitrary datum temperature. The above limit is satisfied if

$$
\theta=-\frac{Q}{2 \pi k} \ln R
$$


The following identities are necessary for further development of the problem

$$
\begin{aligned}
\ln R & =\ln r^{\prime}-\sum_{n=1}^{\infty}\left(\frac{r}{r^{\prime}}\right)^{n} \frac{\cos n\left(\varphi-\varphi^{\prime}\right)}{n}, & & r<r^{\prime} \\
& =\ln r-\sum_{n=1}^{\infty}\left(\frac{r^{\prime}}{r}\right)^{n} \frac{\cos n\left(\varphi-\varphi^{\prime}\right)}{n}, & & r>r^{\prime} .
\end{aligned}
$$

By use of the above identities, eq (3) can be readily shown to be a particular solution to a partial differential equation for heat conduction:

$$
\frac{\partial^{2} \theta_{s}}{\partial r^{2}}+\frac{1}{r} \frac{\partial \theta_{s}}{\partial r}+\frac{1}{r^{2}} \frac{\partial^{2} \theta_{s}}{\partial \varphi}=0, \quad s=1,2
$$

Here, the source is assumed to be contained in region $s=1$, where $c<r<a$; for region $s=2$, $a<r<b$. Solutions to (5) are:

For region $1, c<r<r^{\prime}$

$$
\theta_{1}=\frac{Q}{2 \pi k_{1}}\left[-\ln r^{\prime}+P_{1}+\sum \frac{\cos n\left(\varphi-\varphi^{\prime}\right)}{n}\left\{\left(\frac{r}{r^{\prime}}\right)^{n}+A_{n}\left(\frac{r r^{\prime}}{a^{2}}\right)^{n}+B_{n}\left(\frac{c^{2}}{r r^{\prime}}\right)^{n}\right\}\right] .
$$

For region $1, r^{\prime}<r<a$

$$
\theta_{1}=\frac{Q}{2 \pi k_{1}}\left[-\ln r+P_{2}+\sum \frac{\cos n\left(\varphi-\varphi^{\prime}\right)}{n}\left\{\left(\frac{r^{\prime}}{r}\right)^{n}+A_{n}\left(\frac{r r^{\prime}}{a^{2}}\right)^{n}+B_{n}\left(\frac{c^{2}}{r r^{\prime}}\right)^{n}\right\}\right] .
$$

For region 2

$$
\theta_{2}=\frac{Q}{2 \pi k_{1}}\left[-\ln r+P_{3}+\sum \frac{\cos n\left(\varphi-\varphi^{\prime}\right)}{n}\left\{C_{n} r^{n}+D_{n} r^{-n}\right\}\right]
$$

The constants $P_{1}, P_{2}, P_{3}, A_{n}, B_{n}, C_{n}$, and $D_{n}$ are determined from the boundary conditions:

$$
\begin{aligned}
& \theta_{2}=0 \quad \text { at } r=b \\
& \frac{\partial \theta_{1}}{\partial r}=0 \quad \text { at } r=c \\
& \theta_{1}=\theta_{2} \quad \text { at } r=a \\
& k_{1} \frac{\partial \theta_{1}}{\partial r}=k_{2} \frac{\partial \theta_{2}}{\partial r} \quad \text { at } r=a \text {. }
\end{aligned}
$$

The boundary condition, $\frac{\partial \theta_{1}}{\partial r}=0$ at $r=c$ assumes that the region $0<r<c$ contains a perfect insulator, and does not assume the temperature at $r=c$ to be constant. This boundary condition is a mathematical expediency for the development to the problem of more than one line heat source, where the heat flows directed toward the center from heat sources positioned on $r^{\prime}$ tend to nullify each other at $r=c$. The degree to which the gradient becomes negligible at $r=c$ depends upon the uniformity of the magnitudes and positions of the heat sources. From a practical viewpoint, this boundary condition approximates a system where region 1 is composed of a material of high thermal conductivity and the region $0<r<c$ is a thermal insulator. Otherwise, for the single heat source, the region $0<r<c$, and its thermal conductivity must be introduced into the analysis.

Substitution of the boundary and continuity conditions in eq (6) gives for region 1

$$
\theta_{1}=\frac{Q}{2 \pi k_{1}}\left[\ln a / r^{\prime}+\sigma \ln b / a\right]+\frac{Q}{2 \pi k_{1}} \sum_{n=1}^{\infty} \frac{\cos n\left(\varphi-\varphi^{\prime}\right)}{n}\left[\left(\frac{r}{r^{\prime}}\right)^{n}+\left(\frac{c^{2}}{r r^{\prime}}\right)^{n}+\psi_{n}(r)\right]
$$


for $c<r<r^{\prime}$, where

$$
\begin{aligned}
\psi_{n}(r) & =\frac{(\sigma X-1)\left(\frac{r r^{\prime}}{a^{2}}\right)^{n}}{(\sigma X Z+1)\left[1+\left(\frac{c}{a}\right)^{2 n}\right]}\left[1+\left(\frac{c}{r}\right)^{2 n}+\left(\frac{c}{r^{\prime}}\right)^{2 n}+\left(\frac{c^{2}}{r r^{\prime}}\right)^{2 n}\right] \\
X & =\frac{1-(a / b)^{2 n}}{1+(a / b)^{2 n}} \\
Z & =\frac{1-(c / a)^{2 n}}{1+(c / a)^{2 n}} .
\end{aligned}
$$

For $r^{\prime}<r<a$, interchange $r$ and $r^{\prime}$.

\section{b. Multiple Line Heat Sources}

Consider $m$ continuous line sources of magnitude $Q_{p}$ at $\left(r^{\prime}, \varphi_{p}^{\prime}\right), p=1,2, \ldots, m$. The separate solutions involving $\varphi_{p}^{\prime}$ are additive. Thus,

$$
\theta_{1}=\frac{1}{2 \pi k_{1}}\left[\ln a / r^{\prime}+\sigma \ln b / a\right] \sum_{p=1}^{m} Q_{p}+\frac{1}{2 \pi k_{1}} \sum_{n=1}^{\infty} \frac{\left(\frac{r}{r^{\prime}}\right)^{n}+\left(\frac{c^{2}}{r r^{\prime}}\right)^{n}+\psi_{n}(r)}{n} \sum_{p=1}^{m} Q_{p} \cos n\left(\varphi-\varphi_{p}^{\prime}\right) .
$$

If $Q_{p}=Q$, and $\varphi_{p}^{\prime}=2 \pi p / m$, i.e., equally spaced equal line sources, then

$$
\begin{aligned}
\sum_{p=1}^{m} \cos q\left(\varphi-\frac{2 \pi p}{m}\right) & =\cos q \varphi \Sigma \cos \frac{2 \pi p q}{m}+\sin q \varphi \Sigma \sin \frac{2 \pi p q}{m} \\
& =0 \text { for } q \neq m n \\
& =m \cos m n \varphi \text { for } q=m n,
\end{aligned}
$$

and eq (10) becomes ${ }^{2}$

$$
\theta_{1}=\frac{m Q}{2 \pi k_{1}}\left[\ln a / r^{\prime}+\sigma \ln b / a\right]+\frac{m Q}{2 \pi k_{1}} \sum_{n=1}^{\infty} \frac{\cos q \varphi}{q}\left[\left(\frac{r}{r^{\prime}}\right)^{q}+\left(\frac{c^{2}}{r r^{\prime}}\right)^{q}+\psi_{q}(r)\right]
$$

where $q=m n$.

Using (4), and rearranging, eq (11) becomes valid for the whole region $1, c<r<a$,

$$
\begin{aligned}
\theta_{1}=\frac{Q}{2 \pi k_{1}}\left[m \sigma \ln b / a-\frac{1}{2} \ln \{\right. & \left.\left(\frac{r^{\prime}}{a}\right)^{2 m}+\left(\frac{r}{a}\right)^{2 m}-2\left(\frac{r r^{\prime}}{a^{2}}\right)^{m} \cos m \varphi\right\} \\
& \left.-\frac{1}{2} \ln \left\{1+\left(\frac{c^{2}}{r r^{\prime}}\right)^{2 m}-2\left(\frac{c^{2}}{r r^{\prime}}\right)^{m} \cos m \varphi\right\}+m \Sigma \frac{\cos q \varphi}{q} \psi_{q}(r)\right] .
\end{aligned}
$$

Inspection of (9) shows that the ratios $X, Z$, and the dimension ratios are less than unity, so that the expression $\psi_{q}(r)$, in (12), for $m \geq 2$, becomes quite small with increase in $n$, and only a few terms of the series need be evaluated.

The average temperature, $\bar{\theta}_{r}$, at any radius $r$, can be found by integrating eq (11) with respect to $\varphi$, or

$$
\begin{aligned}
\bar{\theta}_{r} & =\frac{1}{2 \pi} \int_{0}^{2 \pi} \theta_{1} d \varphi=\frac{m Q}{2 \pi k_{1}}[\ln a / r+\sigma \ln b / a], \quad r^{\prime}<r<a \\
& =\frac{m Q}{2 \pi k_{1}}\left[\ln a / r^{\prime}+\sigma \ln b / a\right], \quad c<r<r^{\prime} .
\end{aligned}
$$

\footnotetext{
${ }^{2}$ Equation (11) may also be derived by considering a wedge, $t=r e^{i \varphi}, 0<\varphi<2 \pi / m, c<r<b$, with a line source at $r=r^{\prime}$ lying on its bisector Due to the symmetry of the line sources assume no heat flow across the boundaries of the wedge. Making the transformation $w=t^{m}$ gives a single line source at $r=r^{\prime} m$ and $\varphi^{\prime}=\pi$. Using the single line source solution (8), a transformation from the $w$ to $t$ plane gives (11).
} 
Equation (13) substantiates eq (1), which was arrived at intuitively. Similar integration of (10) with respect to $\varphi$ leaves only the first term of the right side of (10). If it is assumed that $m Q_{\mathrm{av}}=\sum_{p=1}^{m} Q_{p}$, then eq (1) is valid for reasonable variations of the distribution and magnitude of the heat sources on the radius $r=r^{\prime}$ (see discussion following (7)).

For a finite number of sources, it is of interest to investigate the ratio

$$
\beta=\frac{\theta_{c}}{\bar{\theta}_{c}}
$$

at $r=c$, as a function of the angle $\varphi$ for variations in the number of line sources and in $\sigma$, the ratio of thermal conductivity in the first region to that in the second. Numerical solutions are shown in figures 2 and 3, where the radial dimensions were fixed by means of the following ratios: $b / a=5.0, r^{\prime} / a=0.7$, and $c / a=0.2$.

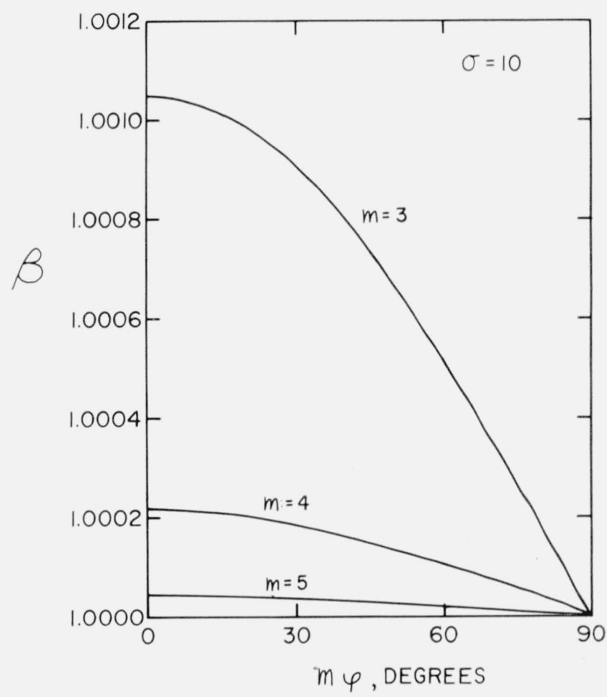

FIGURE 2. Infinite composite cylinder-Ratio $\beta$ eq (14), versus angle for increasing number, $\mathrm{m}$, of line sources, with $\sigma=10, \mathrm{~b} / \mathrm{a}=5, \mathrm{r}^{\prime} / \mathrm{a}=0.7$, and $\mathrm{c} / \mathrm{a}=0.2$.

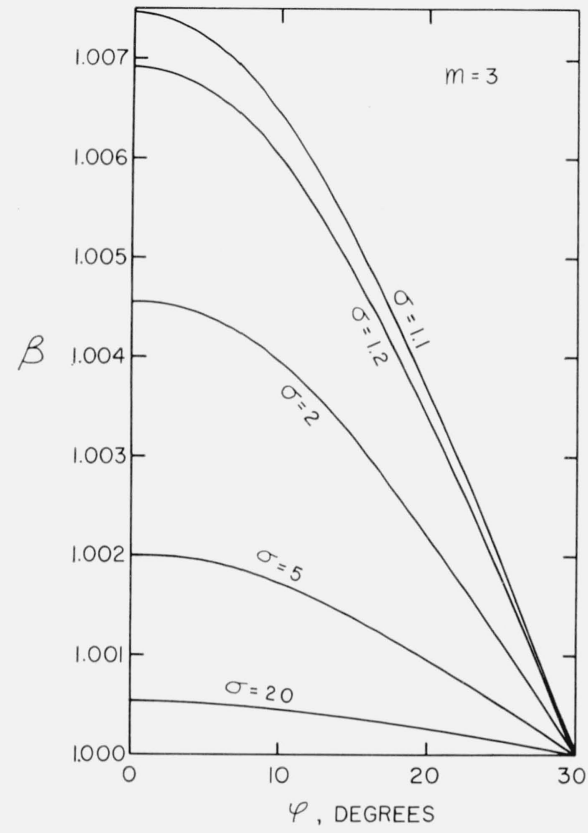

Figure 3. Infinite composite cylinder-Ratio $\beta$ eq (14), versus angle for various values of $\sigma\left(=\mathrm{k}_{1} / \mathrm{k}_{2}\right)$, with $\mathrm{m}=3, \mathrm{~b} / \mathrm{a}=5, \mathrm{r}^{\prime} / \mathrm{a}=0.7$, and $\mathrm{c} / \mathrm{a}=0.2$.

\subsection{Cylinder of Finite Length}

\section{a. Single Line Heat Source}

The heat flux from a continuous line heat source in the finite cylinder $(0<z<l)$. must satisfy the limit (2). A solution that satisfies (2) and the partial differential equation

is

$$
\frac{\partial^{2} \theta}{\partial r^{2}}+\frac{1}{r} \frac{\partial \theta}{\partial r}+\frac{1}{r^{2}} \frac{\partial^{2} \theta}{\partial r^{2}}+\frac{\partial^{2} \theta}{\partial z^{2}}=0
$$

$$
\theta=\frac{2 Q}{\pi^{2} k} \sum_{n=1}^{\infty} \frac{\sin \frac{j \pi z}{l}}{j} K_{0}\left(\frac{j \pi R}{l}\right) \quad j=2 n-1 .
$$


The following identities and definitions are necessary for further development of the problem

$$
\begin{array}{rlrl}
K_{0}(\alpha R) & =\sum_{i=-\infty}^{+\infty} \cos i\left(\varphi-\varphi^{\prime}\right) K_{i}\left(\alpha r^{\prime}\right) I_{i}(\alpha r) & & r<r^{\prime} \\
K_{0}(\alpha R) & =\sum_{i=-\infty}^{+\infty} \cos i\left(\varphi-\varphi^{\prime}\right) K_{i}(\alpha r) I_{i}\left(\alpha r^{\prime}\right) & & r>r^{\prime} \\
F_{n}(x, y) & =I_{n}(x) K_{n}(y)-I_{n}(y) K_{n}(x) & \\
F_{n}^{\prime \prime}(x, y) & =I_{n}(x) K_{n}^{\prime}(y)-I_{n}^{\prime}(y) K_{n}(x) & \\
F_{n}^{\prime \prime}(x, y) & =I_{n}^{\prime}(x) K_{n}^{\prime}(y)-I_{n}^{\prime}(y) K_{n}^{\prime}(x) & \\
F_{n}(x, x) & =F_{n}^{\prime \prime}(x, x)=0 \\
F_{n}^{\prime}(x, x) & =-1 / x .
\end{array}
$$

Where $I_{n}(x)$ and $K_{n}(x)$ are modified Bessel functions of order $n$, and of the first and second kind, respectively.

Proceeding as in section 2.1.a, and using (17), the solutions for the two finite regions of the composite are:

for region $2, a<r<b$

$$
\theta_{2}=\frac{2 Q}{\pi^{2} k_{1}} \sum_{n=1}^{+\infty} \frac{\sin \frac{j \pi z}{l}}{j} \sum_{i=-\infty}^{\infty} M_{i} \cos i\left(\varphi-\varphi^{\prime}\right) \frac{F_{i}\left(\frac{j \pi b}{l}, \frac{j \pi r}{l}\right)}{F_{i}\left(\frac{j \pi b}{l}, \frac{j \pi a}{l}\right)}
$$

for region $1, r^{\prime}<r<a$

$$
\theta_{1}=\frac{2 Q}{\pi^{2} k_{1}} \sum_{n=1}^{\infty} \frac{\sin \frac{j \pi z}{l}}{j} \sum_{i=-\infty}^{+\infty} \cos i\left(\varphi-\varphi^{\prime}\right)\left\{K_{i}\left(\frac{j \pi r}{l}\right) I_{i}\left(\frac{j \pi r^{\prime}}{l}\right)+U_{i}\left(\frac{j \pi r}{l}\right)\right\}
$$

for region $1, c<r<r^{\prime}$

where

$$
\theta_{l}=\frac{2 Q}{\pi^{2} k_{1}} \sum_{n=1}^{\infty} \frac{\sin \frac{j \pi z}{l}}{j} \sum_{i=-\infty}^{+\infty} \cos i\left(\varphi-\varphi^{\prime}\right)\left\{K_{i}\left(\frac{j \pi r^{\prime}}{l}\right) I_{i}\left(\frac{j \pi r}{l}\right)+U_{i}\left(\frac{j \pi r}{l}\right)\right\}
$$

$$
U_{i}\left(\frac{j \pi r}{l}\right)=P_{i} I_{i}\left(\frac{j \pi r}{l}\right)+Q_{i} K_{i}\left(\frac{j \pi r}{l}\right)
$$

$M_{i}, P_{i}$, and $Q_{i}$ must satisfy the boundary and continuity conditions (7). The exterior boundary condition, that $\theta=0$ at the surfaces $z=0, z=l$, and $r=b$, is satisfied by the above equations. For region $1, c<r<r^{\prime}$

$$
\theta_{1}=\frac{2 Q}{\pi^{2} k_{1}} \sum_{n} \frac{\sin \frac{j \pi z}{l}}{j} \sum_{i} \gamma_{i}\left(r^{\prime}, r\right) \cos i\left(\varphi-\varphi^{\prime}\right)
$$

where

$$
\begin{gathered}
\gamma_{i}\left(r^{\prime}, r\right)=\frac{F_{i}^{\prime}\left(\frac{j \pi r}{l}, \frac{j \pi c}{l}\right)\left[F_{i}^{\prime}\left(\frac{j \pi b}{l}, \frac{j \pi a}{l}\right) F_{i}\left(\frac{j \pi r^{\prime}}{l}, \frac{j \pi a}{l}\right)-\sigma F_{i}^{\prime}\left(\frac{j \pi b}{l}, \frac{j \pi a}{l}\right) F_{i}^{\prime}\left(\frac{j \pi r^{\prime}}{l}, \frac{j \pi a}{l}\right)\right]}{\delta_{i}} \\
\delta_{i}=\sigma F_{i}\left(\frac{j \pi b}{l}, \frac{j \pi a}{l}\right) F_{i}^{\prime \prime}\left(\frac{j \pi a}{l}, \frac{j \pi c}{l}\right)-F_{i}^{\prime}\left(\frac{j \pi b}{l}, \frac{j \pi a}{l}\right) F_{i}^{\prime}\left(\frac{j \pi a}{l}, \frac{j \pi c}{l}\right) .
\end{gathered}
$$


Consider, as in section 2.1.b, $m$ equally spaced equal sources at $r=r^{\prime}$. Equation becomes

$$
\theta_{1}=\frac{2 m Q}{\pi^{2} k_{1}} \sum_{n=1}^{\infty} \frac{\sin \frac{j \pi z}{l}}{j}\left\{\gamma_{0}\left(r^{\prime}, r\right)+2 \sum_{i=1}^{\infty} \gamma_{q}\left(r^{\prime}, r\right) \cos q \varphi\right\}
$$

where $q=m i$ and $j=2 n-1$.

It is of particular interest to evaluate the mean temperature at $r=c$ and $z=l / 2$,

$$
\left(\bar{\theta}_{c}\right)_{z=l / 2}=\frac{2 m Q}{\pi^{2} k_{1}} \sum_{n=1}^{\infty} \frac{(-1)^{n+1}}{j} \gamma_{0}\left(r^{\prime}, c\right)
$$

to determine the effects of longitudinal heat flow by the ratio, $\tau$, of this temperature to the average temperature for the infinitely long cylinder at $r=c$ as given by (13):

$$
\tau=\frac{\left(\bar{\theta}_{c}\right)_{z=l / 2}}{\bar{\theta}_{c}}
$$

Numerical solutions for $\tau$ versus the dimension ratio $l / b$ for various values of $\sigma$, are shown in figure 4 for fixed radius dimension ratios $b / a=5.0, r^{\prime} / a=0.7, c / a=0.2$.

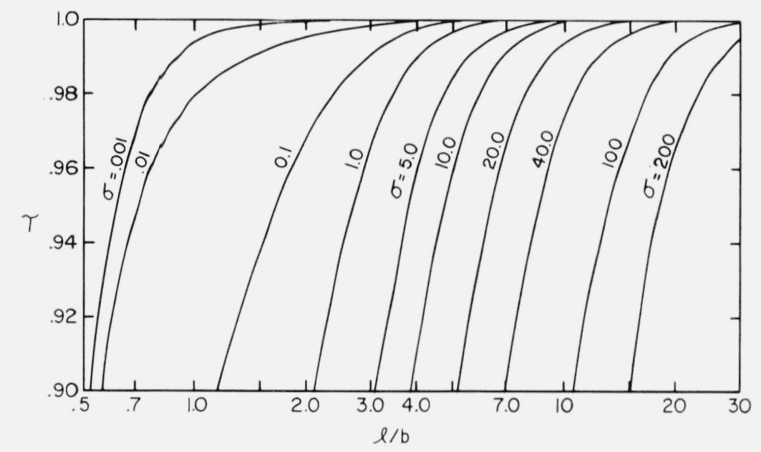

Figure 4. Finite composite cylinder-Ratio $\tau$ eq (21) versus $1 / b$ (cylinder length/outer radius) for various values of $\sigma$, with $\mathrm{b} / \mathrm{a}=5, \mathrm{r}^{\prime} / \mathrm{a}=0.7$, and $\mathrm{c} / \mathrm{a}=0.2$.

\subsection{Special Cases With $\sigma=1$}

An interesting special case of multiple line sources in an infinitely long cylinder is the case where $k_{1}=k_{2}$. Substitution of $\sigma=1$ in (11) gives

where

$$
\theta_{1}=\frac{m Q}{2 \pi k_{1}} \ln b / r^{\prime}+\frac{m Q}{2 \pi k_{1}} \sum \frac{\cos q \varphi}{q}\left[\left(\frac{r}{r^{\prime}}\right)^{q}+\left(\frac{c^{2}}{r r^{\prime}}\right)^{q}-\psi_{q}^{\prime}(r)\right]
$$

$$
\psi_{q}^{\prime}(r)=\frac{1+\left(\frac{c}{r}\right)^{2 q}+\left(\frac{c}{r^{\prime}}\right)^{2 q}+\left(\frac{c^{2}}{r r^{\prime}}\right)^{2 q}}{\left(\frac{b^{2}}{r r^{\prime}}\right)^{q}+\left(\frac{c^{2}}{r r^{\prime}}\right)^{q}}
$$

For $r=c$ and for $c<<r^{\prime}<b$, only the first term in (22) need be considered. Physically, this special case represents a single cylinder of homogeneous material, in which $m$ heater wires parallel to the axis are embedded at a radius $r^{\prime}$ with equal angular spacing. The temperature is measured at the axis of the cylinder.

For the special case in a finite cylinder where $c=0$ and $k_{1}=k_{2}$ or $\sigma=1$, the $\gamma_{i}$ of eq (19) becomes

$$
\gamma_{i}\left(r^{\prime}, r\right)=\frac{I_{i}\left(\frac{k \pi r}{l}\right) F_{i}\left(\frac{k \pi b}{l}, \frac{k \pi r^{\prime}}{l}\right)}{I_{i}\left(\frac{k \pi b}{l}\right)} .
$$




\section{Discussion}

The variations in temperature at a radius $c\left(c<r^{\prime}\right)$ with respect to angle are illustrated in figures 2 and 3, using fixed values of the radius dimension ratios $b / a, r^{\prime} / a$ and $c / a$. Figures 2 and 3 , for the infinitely long cylinder, show that the angular variation decreases with increase in the number of line sources $(m)$, and also with increase in the value of $\sigma=\left(k_{1} / k_{2}\right)$. The latter is intuitively evident since the magnitudes of all temperature differences in the inner cylinder obviously are reduced as its conductivity increases relative to that of the outer cylinder.

Figure 4 shows the above-datum temperature at the center and midpoint of a cylinder of finite length as a fraction of the corresponding temperature attained in an infinitely long cylinder (eq (1)), for variations of $\sigma$ and of $l / b$, the ratio of the cylinder length to the outside radius of the outer cylinder. Since this analysis assumes that the ends of the two concentric cylinders are maintained at the same temperature $(\theta=0)$ as the outer surface at $r=b$, the $l / b$ ratio necessary to maintain a specified $\tau$-value from figure 4 may be somewhat different from that encountered in most applications. With an increasing value of $\sigma$, longitudinal heat flow in the inner cylinder increases relative to the radial heat flow, hence it is necessary to increase the cylinder length to avoid an increase in the error. For values of $\sigma$ much less than unity, the length of cylinder necessary to restrict the error is controlled chiefly by the effect of longitudinal heat flow to the ends of the outer cylinder.

This analysis confirms that for steady radial heat flow in angularly isotropic infinitely Jong circular cylinders, the temperature at the axis is equal to the average temperature over any coaxial cylindrical surface which does not envelop a heat source. Thus, in such a cylindrical system having steady line heat sources parallel to the axis and all at a radius $r^{\prime}$, the temperature at the axis is equal to the average temperature at the cylindrical surface of radius $r^{\prime}$. If the line sources were uniformly spaced angularly, there would be no difference in average temperature or heat flux at a radius greater than $r^{\prime}$, whether the heat was supplied by the distributed line sources and the average temperature at $r^{\prime}$ was determined by a measurement at the axis, or whether the same aggregate heat was supplied at the axis, and the temperature at $r^{\prime}$ was determined by averaging the temperatures measured at the several angular positions at $r^{\prime}$. In short, the axial position and the equiangular positions at $r^{\prime}$, respectively, can be used interchangeably as sites for heat supply or temperature measurement, as convenient, without affecting the average heat flow pattern in the cylinder beyond $r^{\prime}$.

The use of equiangularly distributed line sources for heat supply has advantages in some applications. For example, the apparatus described by Flynn [2] used a cylindrical ceramic core as a central heat source in testing a hollow cylinder of fine granular material. If the granular material were very coarse, the thermal contact between the granular particles and the smooth convex ceramic surface might be enough different from that between particles to cause an unwanted and not easily ascertainable temperature drop in the critical region near the ceramic core. If, on the other hand, such a coarse granular material were tested as a complete cylinder, with temperature measured at the axis and line heat sources at radius $r^{\prime}$ in the material (i.e., the case of $\sigma=1$, eq (22)), the oddities of thermal contact of particles with the line heat source would be inappreciable at short distances from the line sources, since the heat flow there would be that occurring in the particulate region.

Another example, of possible experimental interest, is that of conducting a measurement of the thermal conductivity of a solid by the Powell "stacked-disk" method [3], but using" the axial hole for the temperature measurement and the inner circle of holes (preferably three or more) for heat supply, in reverse of the arrangement used by Powell and by others. In this way, uncertainties arising from measurements of temperature in regions of large temperature gradient, or due to disturbances of the heat flow pattern by the thermocouples, would be largely avoided. 
The author is indebted to J. C. Jaeger, Professor of Geophysics in the Australian National University, for his comments on an earlier draft of this paper.

\section{References}

[1] H. S. Carslaw and J. C. Jaeger, Conduction of heat in solids, 2d ed., pp. 385, 423 (Oxford University Press, London, 1959).

[2] D. R. Flynn, A radial flow apparatus for determining the thermal conductivity of loose-fill insulations to high temperatures, J. Research NBS 6\%C (Eng. and Instr.) No. 2, 129-137 (Apr.-June 1963).

[3] R. W. Powell, Further measurements of the thermal and electrical conductivity of iron at high temperatures, Proc. Phys. Soc. 51, p. 411 (London, 1939).

(Paper 67C2-125) 